\title{
EL USO DE LOS ENCINOS EN LA REGIÓN DE la Malinche, Estado de Tlaxcala, México
}

\author{
María Mercedes A. Espejel Rodríguez ${ }^{1}$, Noé Santacruz García ${ }^{1}$ \\ y MOISÉS SÁNCHEZ FLORES ${ }^{2}$
}

\begin{abstract}
IInvestigadores del Centro de Investigaciones Interdisciplinarias sobre el Desarrollo Regional (CIISDER), Universidad Autónoma de Tlaxcala. Boulevard del Maestro No. 902, Col. La Loma Xicohténcatl, Tlaxcala, Tlax. C.P. 90070. Teléfono y Fax 01-246-27380. E-mail ciisder@cci.uatx.mx. ${ }^{2}$ Licenciado en Biología Agropecuaria, Universidad Autónoma de Tlaxcala. ${ }^{3} \mathrm{La}$ información presentada fue obtenida para el proyecto "La importancia y deterioro del recurso bosque en la región de la Malinche, estado de Tlaxcala" financiado por SIZA-CONACYT
\end{abstract}

\begin{abstract}
Resumen. Con base en la revisión de ejemplares de herbario se identificaron 10 especies de encinos (Quercus) en la región de La Malinche, Tlaxcala. Se presenta un listado de las especies y un mapa de su distribución en la región. Se aplicó un cuestionario a través de una entrevista directa a los habitantes de nueve localidades asentadas en la zona, para conocer las formas de aprovechamiento de los recursos. A partir de los resultados, se hace un análisis de la importancia socioeconómica que tienen estos elementos. Entre los principales usos detectados se tiene la utilización como combustible (leña y carbón), la obtención de madera, el empleo de la hojarasca como abono orgánico, el uso de la corteza con fines medicinales.

Palabras clave: Quercus, encinos, La Malinche, Tlaxcala, aprovechamiento forestal.

Abstract. Based on the review of herbarium specimens ten species of oaks (Quercus) were identified in the region of La Malinche, Tlaxcala, Mexico. A list of species and a distribution map of each one of them in the region is presented. A survey, through a direct interview, on to the form of utilization of the resource was conducted among the inhabitants of nine localities stablished in the zone. An analysis of the socioeconomic importance of these elements is made. Among the principal uses detected are the utilization as fuelwood and coal, wood, employment of the verbiage as organic fertilizer, and use of the bark as medicine.
\end{abstract}

Key words: Quercus, oak, La Malinche, Tlaxcala, Forestry.

os bosques de encino tienen una distribución muy amplia en climas templados y fríos. En México, los encinares son el segundo grupo importante, por su abundancia, después de los pinares (Zavala 1990). $\mathrm{Al}$ analizar su distribución llaman la atención por ser fisonómicamente dominantes en la vegetación forestal de nuestro país, y por poseer una diversidad de alrededor de 150 especies, distribuidas principalmente en los sistemas montañosos de la República (Rzedowsky, 1978).

Este tipo de vegetación se presenta desde el nivel del mar hasta los $3100 \mathrm{msnm}$; cubre aproximadamente el $95 \%$ del terreno entre los 1200 y $2800 \mathrm{msnm}$. Ocupa aproximadamente el $5 \%$ de la superficie total del país (Flores, 1971. Citado por Rzedowsky, 1978). El género predominante en este tipo de vegetación es Quercus, caracterizado por su gran adaptación a diferentes condiciones del medio, como son relieve, altitud, pendiente, tipo de roca, suelo y otros.

En el estado de Tlaxcala, los bosques de encino ocupan alrededor del $5 \%$ de la superficie de la entidad (INEGI, 1982). Prosperan desde los 2200 hasta los $3000 \mathrm{msnm}$, sobre suelos de origen volcánico, de profundos a someros, en áreas con precipitación anual de 700 a $1000 \mathrm{~mm}$ (Acosta et al., 1991). Estas características les permiten ocupar gran variedad de hábitats, como los que se presentan en el volcán La Malinche, como una estructura geológica que domina el paisaje tlaxcalteca y que abarca aproximadamente el $23 \%$ de la superficie de la entidad y alberga a casi 
la tercera parte de la población estatal, lo que la hace una de las zonas forestales más explotadas, ya que el uso desmesurado y frecuentemente anárquico de los ecosistemas ha propiciado la disminución y muchas veces la desaparición de la vegetación natural.

Con base en el panorama descrito y dada la importancia que tienen los bosques de esta región del estado de Tlaxcala, el presente trabajo tiene como objetivo conocer las especies del género Quercus que se encuentran en La Malinche, su distribución dentro de la zona y la importancia socioeconómica y ecológica que tienen para los habitantes de la región.

\section{Área de estudio}

El volcán La Malinche, Malintzi o Matlalcuéyetl, se localiza entre los $19^{\circ} 05^{\prime}$ y $19^{\circ} 22^{\prime}$ de latitud norte y los $97^{\circ} 53^{\prime}$ y $98^{\circ} 12^{\prime}$ de longitud oeste, en la porción sureste de Tlaxcala; abarca poco más de $30 \mathrm{~km}$ de diámetro y se eleva de los 2300 a los $4461 \mathrm{msnm}$. Político-administrativamente el volcán queda comprendido en $4 / 5$ partes de su superficie dentro de la entidad tlaxcalteca, el resto corresponde al estado de Puebla (Hernández, 1992). Esta montaña es considerada una de las más viejas de la cordillera Neovolcánica, ya que al parecer nació hacia el final del Mioceno, además, se le considera la montaña aislada más importante del país (Sánchez de Tagle, 1978).
La información relacionada con el aprovechamiento de los encinares se obtuvo de las siguientes localidades: Ixtenco, Francisco Javier Mina, Guadalupe Altamira, Los Pilares, San José Teacalco, San Francisco Tetlanohcan, San Rafael Tepatlaxco, Acxotla del Monte y San Isidro Buensuceso. Todas ellas localizadas dentro del área definida como Parque Nacional, el cual fue creado por Decreto Presidencial el 21 de septiembre de 1938 y publicado en el Diario Oficial el 6 de octubre del mismo año.

\section{Metodología}

Para la realización de esta investigación se planteó desarrollar el trabajo en dos etapas. La primera de ellas permitió la identificación de las especies del género Quercus que forman parte de los encinares de La Malinche. En la segunda se obtuvo información sobre de la forma de utilización del recurso encinos y de la importancia que tiene para los habitantes de las localidades asentadas en la falda de la montaña.

Para la identificación de las especies de encinos que forman parte de la cubierta vegetal de La Malinche, se realizaron recorridos por el área para detectar las unidades de vegetación en que existían encinos, se colectaron muestras botánicas que fueron herborizadas y posteriormente comparadas con claves para lograr su determinación. También se tomaron datos de lo-

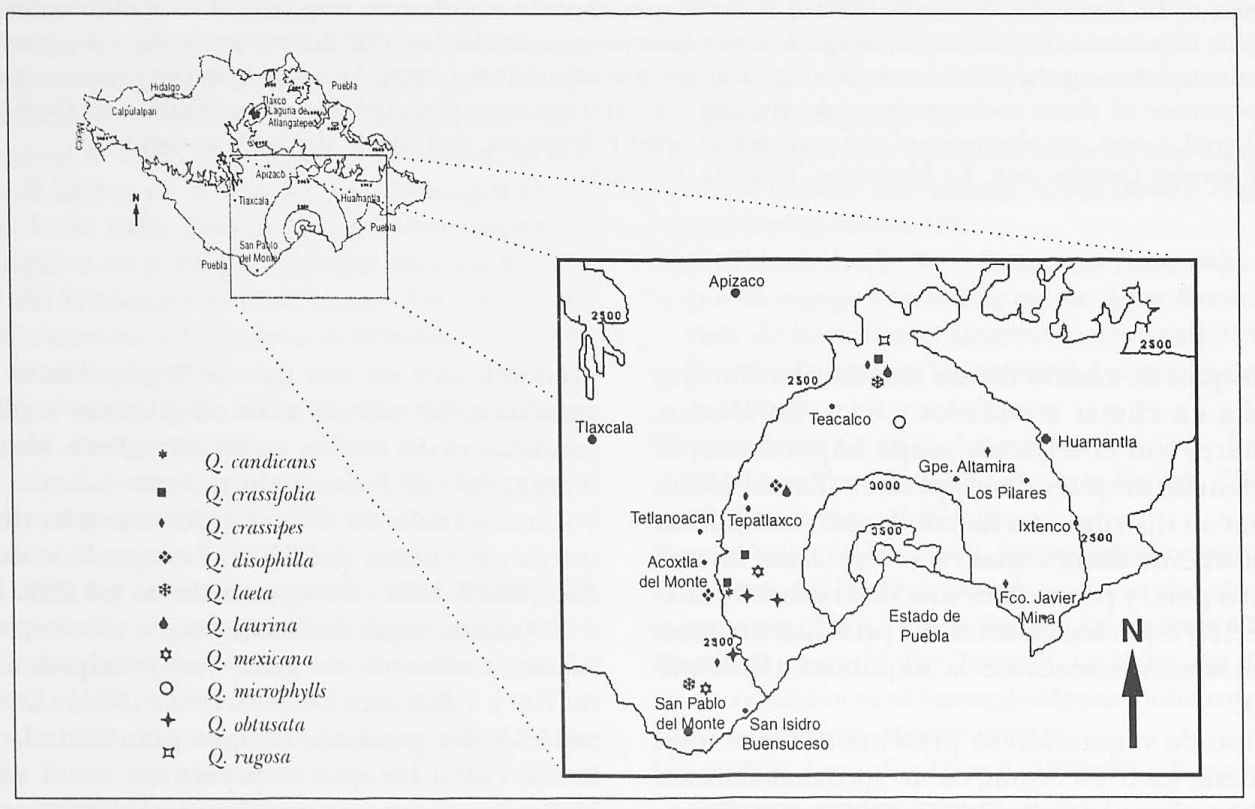

Figura 1. Sitios de colecta de las especies de encinos identificadas en la región de La Malinche, estado de Tlaxcala. 
calización que permitieran establecer y representar de manera gráfica la distribución de las diferentes especies.

La información sobre el uso de los encinos en la región fue obtenida a través de la aplicación de un cuestionario, mediante entrevista directa, a 429 habitantes de nueve localidades de la zona, en el que se les preguntó acerca de los beneficios obtenidos, forma de aprovechamiento y diversos aspectos sobre el deterioro que sus actividades pueden ocasionar al bosque Algunas de las preguntas que se hicieron fueron: de los árboles que hay en el bosque, ¿̇cuál es el que utiliza con mayor frecuencia?, ¿qué productos obtiene de los árboles que hay en la Malinche?, ¿para qué utiliza estos productos? Si se destinan al hogar, ¿para qué se utilizan?, Si se venden los productos, ¿donde se realiza la venta? ¿qué productos se venden con mayor frecuencia?, ¿̇a qué precio los vende?, ¿cada cuando va al bosque a colectar estos productos?, de las actividades que realiza, ¿cuáles dañan al bosque?

\section{Resultados y discusión}

1. Los encinos de La Malinche. A partir de la colecta y revisión de 54 ejemplares de herbario correspondientes al género Quercus procedentes de 12 sitios de colecta de la región de La Malinche, depositados en las colecciones de diferentes instituciones, se identificaron 10 especies de encinos presentes en el área (Santacruz, 1996) :

\section{Quercus candicans Née \\ Q. crassifolia $\mathrm{H}$. \& $\mathrm{B}$. \\ Q. crassipes $\mathrm{H}$. \& B. \\ Q. dysophylla Benth. \\ Q. laeta Liebm. \\ Q. laurina H. \& B. \\ Q. mexicana $\mathrm{H} . \& \mathrm{~B}$. \\ Q. microphylla Née \\ Q. obtusata $\mathrm{H}$. \& B. \\ Q. rugosa Née}

Por su fisonomía, los encinos de La Malinche se caracterizan por ser de talla intermedia, alcanzando alturas entre 3 y $15 \mathrm{~m}$, aunque se encontraron formas arbustivas como es el caso de Quercus microphylla. El crecimiento de estos árboles es lento, alcanzan larga vida y sus fustes son generalmente ramificados a poca altura y torcidos, de ahí que se les considere poco importantes desde el punto de vista forestal.

Respecto a su distribución, estas especies ocupan una gran variedad de ambientes, pudiéndose encontrar principalmente en laderas y barrancas, sobre suelos muy variados. En la figura 1 se muestra la lo-

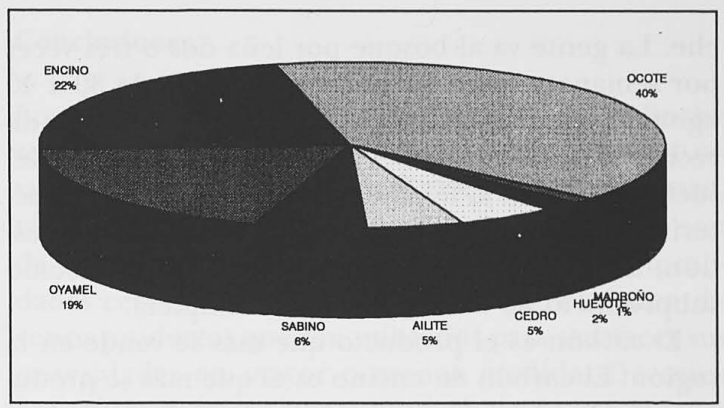

Figura 2. Especies arbóreas mas utilizadas para la obtención de productos en la región de la Malinche (Espejel, 1998).

calización de los sitios de colecta y de cada una de las especies identificadas.

2. Aprovechamiento de los bosques de encino en La Malinche. Los bosques de encino son un recurso de gran importancia para los habitantes aledaños a la Malinche, preguntar sobre el uso más frecuente de árboles, se encontró que los encinos son el segundo grupo de plantas más aprovechado por la población de la región, después de los pinos (figura 2). Los encinos representan un recurso importante por el potencial de su madera, raíces, corteza, hojas y frutos; además de su importancia ecológica, ya que son buenos formadores de suelo y lo protegen de la erosión, albergan y alimentan animales silvestres, soportan epífitas, forman asociaciones micorrícicas, son inductores de un mayor desarrollo biológico de algunas especies de pinos, y proporcionan áreas escénicas y de recreación.

Con las respuestas obtenidas durante la aplicación de cuestionario a los habitantes de La Malinche, se identificaron los usos dados a este recurso y por tanto la importancia socioeconómica que representa para ellos. Se encontró que por la variedad de productos obtenidos por especie, se encuentran ubicados en tercer lugar de importancia, por debajo de los pinos y del oyamel; en la figura 3 se observa la frecuencia de obtención de diversos productos expresada en porcentaje, destaca el hecho que en los porcentajes de obtención de carbón y tierra, los encinos superan a los otros grupos de plantas.

La madera de estos árboles, por sus características biológicas y tecnológicas como dureza y resistencia, es empleada principalmente para la obtención de combustible (leña y carbón). La leña es utilizada básicamente para cocinar sus alimentos y para calentar el baño de temazcal que es muy común en las localidades que se encuentran en la región de La Malin- 
che. La gente va al bosque por leña dos o tres veces por semana y traen de una a dos cargas de 30 a 40 $\mathrm{kg}$. aproximadamente, la mayor parte es cortada directamente de los árboles y muy poca es recogida del suelo. Prefieren la leña de los encinos por sus características de dureza y mayur duración en la combustión, además de que pueden obtener carbón como subproducto de la combustión incompleta.

El carbón es el producto que más se vende en la región. El carbón de encino es el que más se produce y el más cotizado en el estado de Tlaxcala, ya que la gente lo prefiere porque es más duradero y su poder calorífico es mayor, aún cuando su precio es más elevado en comparación con el de otros tipos de carbón que elaboran en la región. El precio varía según la localidad ya que fluctúa actualmente (1998) entre $\$ 2.00$ y $\$ 3.00$ por kilogramo (al momento de realizar las entrevistas, 1996, los precios captados fueron de entre $\$ 1.50$ y $\$ 2.00$ ).

Por medio de las entrevistas se detectó la existencia de personas cuya única actividad es la de carbonero, por lo que su ingreso económico principal proviene del bosque. Otras personas hacen carbón para obtener ingresos adicionales y ayuda en la economía familiar. La importancia económica del carbón queda de manifiesto al encontrar que en seis de las nueve localidades estudiadas se encontraron personas que se dedican a la venta de este producto.

La mayor parte de quienes fabrican carbón, compran los árboles a los dueños de los encinares. El precio de los árboles fluctúa entre $\$ 60.00$ y $\$ 80.00$, dependiendo del tamaño. De cada árbol se pueden obtener entre 20 y $50 \mathrm{~kg}$. de carbón.

La técnica de fabricación es tradicional. Una vez seleccionado el árbol se corta en trozos de tamaño más o menos uniforme, se limpia el terreno y se cla- van estacas que servirán para detener la leña, que se acomoda en forma de cono. Se le cubre con hojas, ramas, pencas de maguey y tierra, dejando algunos respiraderos, se le enciende fuego y se mantiene de 8 a 15 días. Una vez concluida la combustión, se desarma el horno, se enfría el carbón con tierra húmeda y se le envasa en costales para transportarlo a la localidad.

Cabe resaltar que al talar el árbol, se mantiene el tocón, ya que esto favorece el rebrote, con lo que se acorta el tiempo de espera para un nuevo aprovechamiento. Es común observar en la zona de estudio árboles con fustes múltiples, lo que da indicio de esta práctica.

La madera de encino es utilizada de forma local para la elaboración de manceras de arado, cabos para hachas, zapapicos, hoces y palas, ya que su dureza le confiere una mayor resistencia al impacto y una mayor durabilidad en el trabajo; también se emplea, en la fabricación de muebles rústicos y en la obtención de elementos para construcción rural como horcones y morillos, aunque en menor medida, debido a las dificultades para su trabajo y secado y a que sus fustes son cortos y generalmente torcidos.

La hojarasca y el humus producido en los bosques de encino es utilizado como abono orgánico y es muy frecuente ver cómo en los mercados y tianguis de la entidad se vende "tierra para macetas" o "tierra de encino", que es muy solicitada por las amas de casa ya que la utilizan para sus macetas y jardines, el precio oscila entre 10 y 15 pesos el costal. Asimismo en algunas localidades se incorpora a los terrenos de cultivo con el propósito de mejorar la fertilidad del suelo y obtener mejores cosechas.

Es importante resaltar que los entrevistados consideran que la obtención de tierra o de abono es un

Cuadro 1. Usos de los encinos en la región de La Malinche, estado de Tlaxcala

\begin{tabular}{|c|c|c|c|c|c|c|}
\hline \multirow[b]{2}{*}{ Especie } & \multicolumn{6}{|c|}{ Uso regional } \\
\hline & Leña & Carbón & $\begin{array}{l}\text { Implem. } \\
\text { Agricolas }\end{array}$ & $\begin{array}{c}\text { Construc. } \\
\text { Rural }\end{array}$ & Medicinal & Forraje \\
\hline \multicolumn{7}{|l|}{ Q. candicans } \\
\hline Q. crassifolia & $x$ & $x$ & & & & \\
\hline Q. crassipes & $x$ & $x$ & & & $x$ & $x$ \\
\hline Q. dysophylla & $x$ & & $x$ & & & \\
\hline Q. laeta & $x$ & $x$ & & & & \\
\hline Q. laurina & $x$ & $x$ & & $x$ & & \\
\hline Q. mexicana & $x$ & $x$ & & $x$ & & \\
\hline \multicolumn{7}{|l|}{ Q. microphylla } \\
\hline Q. obtusata & $x$ & $x$ & & $x$ & $x$ & $x$ \\
\hline Q. rugosa & $x$ & & & $x$ & & \\
\hline
\end{tabular}




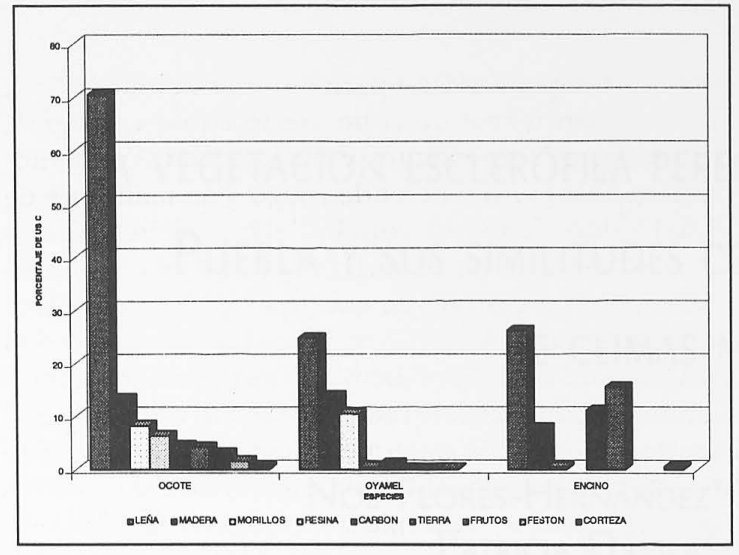

Figura 3. Principales productos obtenidos de las especies más utilizadas.

problema que está ocasionando deterioro del bosque, ya que se sacan grandes cantidades de tierra, principalmente los habitantes de San José Teacalco, San Isidro Buensuceso, San Francisco Tetlanohcan e Ixtenco, que son los principales vendedores de este producto. Los informantes consideran que el saqueo ha deteriorado el bosque porque junto con la hojarasca se extraen semillas y plantas jóvenes, con lo que se disminuye la regeneración natural; además de señalar la sobreexplotación.

El uso de los encinos como plantas medicinales también es conocido en la región, por ejemplo la corteza es empleada para el endurecimiento de las encías y en la preparación de tintes y tratamientos para la caída del cabello.

Otros usos identificados en algunas zonas de La Malinche son: las hojas frescas de estos árboles son empleadas como forraje no convencional, principalmente para alimentar burros, esta información había sido previamente reportada por Rodríguez (1990). En otras regiones del país también se le da este uso ( $\mathrm{Za}$ vala, 1990), empleando las hojas para alimentar cabras y los frutos para alimentar cerdos.

Los habitantes de algunas de las localidades estudiadas recolectan los frutos para ser utilizados en la elaboración de adornos navideños, los cuales son vendidos en los mercados locales durante el mes de diciembre.

En el cuadro 1 se presentan los usos identificados para cada una de las especies que se distribuyen en La Malinche.

\section{Conclusiones}

Los bosques de encino de la región de La Malinche son de gran importancia para los habitantes del estado de Tlaxcala por los beneficios que proporcionan, tanto ecológicos como económicos, sin embargo son de mayor relevancia para los habitantes de las localidades cercanas a estos bosques, ya que obtienen diversos productos que son utilizados para satisfacer sus necesidades en mayor o menor medida. De estos productos destacan por su frecuencia de uso la leña, madera, carbón y abono, los cuales son empleados principalmente para el consumo doméstico y, en ocasiones, para venderlos.

\section{Bibliografía}

Acosta R., Delgado J. L. y Cervantes P. 1991. La vegetación del estado de Tlaxcala, México. Folleto No. 6. Jar * n Botánico Tizatlán-Gobierno del estado de Tlaxcala. 31 pp

Espejel Rodriguez, Ma. Mercedes A. 1998. La importancia y deterioro del recurso bosque en la región de la Malinche, estado de Tlaxcala. Tesis Maestría en Análisis Regional. CIISDERMAR Universidad Autónoma de Tlaxcala. 194 pp.

Espinosa J. 1979. Fagaceae. En: Rzedowki y Rzedowski (compiladores) Flora fanerogámica del Valle de México, Vol. I. CECSA, México. pp 104-114

Hernández L., Acosta R. y Galindo G. 1992. Los muérdagos enanos (Arceuthobium spp) en los bosques de pinos del volcán La Malinche, estado de Tlaxcala. Folleto No. 15. Jardín Botánico Tizatlán-Gobierno del estado de Tlaxcala. 55 pp.

INEGI. 1982. Sintesis Geográfica del estado de Tlaxcala. Secretaría de Programación y Presupuesto. México.

Rodríguez, M. 1990. Estudio de la flora útil de los estados de Puebla y Tlaxcala. I encuentro regional de investigadores de flora y fauna. Zona $\mathrm{V}$ de ANUIES. Tlaxcala, p 78.

Rzedowski J. 1978. Vegetación de México. LIMUSA, México.

Sánchez de Tagle, G. 1978. Contribución al conocimiento de la fauna herpetológica del Parque Nacional La Malinche. Tesis Biólogo. Facultad de Ciencias, México. UNAM, 119 pp.

Santacruz N. 1996. Estudio Florístico Taxonómico del género Quercus en el estado de Tlaxcala, México. Tesis Biólogo. Departamento de Agrobiología, Universidad Autónoma de Tlaxcala. 119 pp.

Zavala F. 1989. Identificación de encinos de México. División de Ciencias Forestales. Universidad Autónoma Chapingo, México. 150 pp.

Zavala F. 1990. Los encinos mexicanos, un recurso desaprovechado. Ciencia y Desarrollo Vol. XVI, Num. 95. CONA. CyT, México. pp 43-51. 